\title{
DOSAGE COLORIMÉTRIQUE \\ DU PALMITATE D'ASCORBYLE DANS LES MATIËRES GRASSES
}

\author{
par \\ J. BUDSLAWSKI et $\mathrm{K}$. POGORZELSKI \\ Laboratoire de chimie du lait et des produits laitiers \\ à l'école supérieure d'agriculture, Olsztyn, (Pologne)
}

\section{I. - Introduction}

Le palmitate d'ascorbyle (PA), produit liposoluble d'esterification de l'acide L-ascorbique avec de l'acide palmitique [1], qui garde ses propriétés réductrices de l'acide L-ascorbique, est souvent employé en qualité d'antioxygène pour les huiles et produits gras [2, 3]. Sa formule de constitution est la suivante:<smiles>CCCC(=O)OCC(O)C1OC(=O)C(O)=C1O</smiles>

$1 \mathrm{mg}$ de palmitate d'ascorbyle correspond à $0,425 \mathrm{mg}$ d'acide L-ascorbique en valeur biologique.

Jusqu'à présent, à notre connaissance, il n'y avait pas de méthode appropriée au dosage du PA; mais il est possible de l'identifier par voie chromatographique [4]. La méthode colorimétrique de dosage de l'acide L-ascorbique, décrite par SchmalL et collaborateurs [5], nous a donné l'idée de chercher à l'adapter au dosage du PA. Cette méthode repose sur une réaction qui a lieu entre l'acide L-ascorbique et 4-méthoxy-2-nitroaniline diazée en milieu acide, donnant ensuite naissance à un composé coloré en milieu alcalin, ayant un maximum d'absorption à $570 \mathrm{~m} \mu$ de longueur d'onde. La marche de cette réaction selon Schmall et al. [6] est la suivante :<smiles>COc1ccc(NNC(=O)C(=O)OC2COCC2C(=O)OCc2ccc(N=NNC3C(O)=C(O)C(=O)OC3CO)c([N+](=O)[O-])c2)cc1</smiles> 


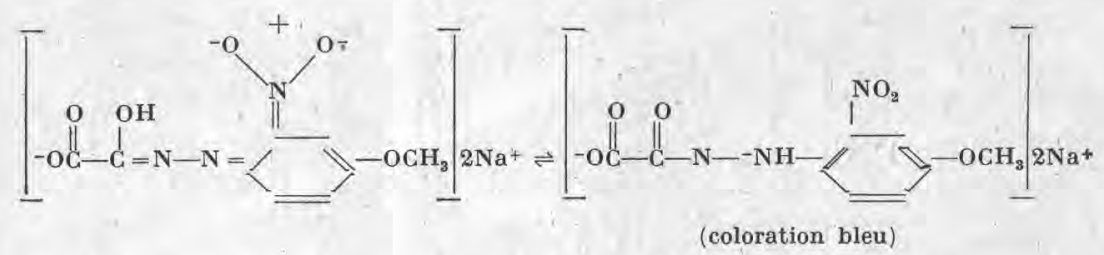

Étant donné que le PA garde ses liaisons diénoliques intactes, il est possible qu'il soit apte à entrer en réaction avec le réactif diazé, ce que nous avions essayé avec succès en utilisant la technique de Schmall [5].

\section{II. - Desoription de la technique}

a) Réactifs.

1. Réactif aminé : $500 \mathrm{mg}$ de 4-méthoxy-2-nitroaniline fraîchement récristallisée (p.f. $125^{\circ} \mathrm{C}$ ), dans l'éthanol; on dissout dans $125 \mathrm{ml}$ d'acide acétique glacial et l'on dilue à $250 \mathrm{ml}$ avec de l'acide sulfurique (dilué à 10 p. 100). Le réactif est stable à la température du laboratoire pendant un mois.

2. Solution aqueuse de $\mathrm{NaNO}_{2}$ à 2 p. 100.

3. Ethanol (on peut utiliser aussi l'alcool isopropylique ou n-butylique).

4. Solution aqueuse de $\mathrm{NaOH}$ à 10 p. 100.

5. Solution aqueuse d'acide oxalique à 0,5 p. 100.

6. Ether éthylique libre de peroxydes.

7. Solution standard de palmitate d'ascorbyle : $50 \mathrm{ml}$ de solution éthanolique contenant $50 \mathrm{mg}$ de PA; on la prépare avant l'utilisation. Il est recommandé de préparer la solution standard avec de l'alcool contenant 0,5 p. 100 d'acide oxalique, ce qui prolonge sa stabilité.

b) Mode opératoire.

Dans un bécher de $100 \mathrm{ml}$ de capacité on mélange $1 \mathrm{ml}$ de réactif aminé (réactif 1 ) avec $1 \mathrm{ml}$ de solution de nitrite de sodium (réactif 2) jusqu'à disparition de la coloration, puis on ajoute $30 \mathrm{ml}$ d'alcool (réactif 3 ), on mélange et on ajoute de nouveau $3 \mathrm{ml}$ d'acide oxalique (réactif 5) et une quantité appropriée d'échantillon de graisse contenant le PA à doser (la quantité de PA dans l'échantillon ne doit pas dépasser $2,5 \mathrm{mg}$ ). Après dissolution complète de l'échantillon, on transvase le contenu du bécher sans perte dans une ampoule à brome de $300 \mathrm{ml}$ de capacité et on l'alcalinise avec $10 \mathrm{ml}$ d'hydroxyde de sodium (réactif '4). 
Après le mélange du contenu on ajoute $60 \mathrm{ml}$ d'éther (réactif 6), on secoue et on laisse l'ampoule à brome au repos. Ensuite on soutire la couche inférieure du liquide dans un ballon jaugé de "100 $\mathrm{ml}$ de capacité et on lave la couche éthérée à trois reprises avec de l'hydroxyde de sodium en utilisant pour cela chaque fois $2 \mathrm{ml}$ de lessive. A chaque reprise, après le repos, on soutire la couche inférieure du liquide et l'on réunit au liquide dans le ballon ; ensuite on remplit le ballon avec de l'eau distillée jusqu'au trait de jauge; on mélange et on mesure la densité optique du liquide à $570 \mathrm{~m} \mu$ de longueur d'onde dans un spectrophotomètre. En même temps on procède à un essai à blanc et on détermine la densité optique de deux échantillons standard A et B que l'on prépare avec de la graisse pure additionnée d'une quantité connue de PA à partir de solution standard (réactif 7), en s'arrangeant de manière que l'échantillon A contienne moins de PA que l'échantillon B, et tous les deux moins que l'échantillon étudié. On traite les échantillons standard de la même manière que l'échantillon de la matière grasse étudiée.

Les quantités de réactifs aminé et azoteux ne doivent pas dépasser l'un l'autre $2 \mathrm{ml}$, de plus grandes quantités pouvant causer un abaissement de l'intensité de la coloration du liquide [5]. Un excès d'acide oxalique peut troubler la solution.

c) Calcul des résultats.

Pour calculer les résultats, on peut soit tracer une courbe standard de densité optique en fonction de la concentration du PA dans un échantillon, soit en utilisant la formule que voici :

$$
\mathrm{X}=\mathrm{A}+\frac{(\mathrm{B}-\mathrm{A})(\mathrm{E}-\mathrm{C})}{\mathrm{D}-\mathrm{C}}
$$

où :

$$
\begin{aligned}
& \mathrm{X}=\text { la quantité de PA en mg dans l'échantillon } \\
& \mathrm{A}=\quad-\quad-\quad-\quad-\quad \text { - standard A; } \\
& \mathrm{B}=\quad-\quad-\quad-\quad-\quad-\text { standard B; } \\
& \mathrm{C}=\text { densité optique du standard A ; } \\
& \mathrm{D}=-\quad-\quad-\mathrm{B} \text {; } \\
& \mathbf{E}=\quad-\quad \text { - de l'échantillon dosé. }
\end{aligned}
$$

\section{III. - Résultats et discussion}

a) Précision de la méthode.

On a vérifié la précision de la méthode avec des échantillons de beurre et d'huile de lin additionnés du PA; on a soumis les résultats à l'analyse statistique. 
Préparation de l'échantillon : à un échantillon pesé de la matière grasse on additionnait une quantité connue de PA (1) et ensuite on le dissolvait dans un mélange d'éther et d'éthanol (1:1), on en prélevait des échantillons contenant une quantité déterminée de PA $(0,5$ à $2,5 \mathrm{mg})$ et l'on procédait à un dosage selon la technique décrite plus haut.

\section{TABLEAU I}

DÉTERMINATION DU PALMITATE D'ASCORBYLE DANS LE BEURRE ET L'HUILE DE LIN (en mg)

\begin{tabular}{|c|c|c|c|c|c|c|c|c|}
\hline \multirow[b]{2}{*}{$\begin{array}{l}\text { Echan- } \\
\text { tillons }\end{array}$} & \multicolumn{8}{|c|}{ Palmitate d'ascorbyle } \\
\hline & $\begin{array}{l}\text { addi- } \\
\text { tionné }\end{array}$ & trouvé & $\begin{array}{l}\text { addi- } \\
\text { tionné }\end{array}$ & trouvé & $\begin{array}{l}\text { addi- } \\
\text { tionné }\end{array}$ & trouvé & $\begin{array}{l}\text { addi- } \\
\text { tionné }\end{array}$ & trouvé \\
\hline 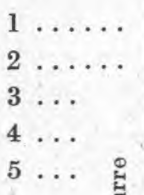 & 1,06 & $\begin{array}{l}1,02 \\
1,04 \\
1,04 \\
1,06 \\
1,05\end{array}$ & 1,17 & $\begin{array}{l}1,17 \\
1,17 \\
1,18 \\
1,16 \\
1,16\end{array}$ & 1,29 & $\begin{array}{l}1,30 \\
1,30 \\
1,30 \\
1,29 \\
1,28\end{array}$ & 1,84 & $\begin{array}{l}1,81 \\
1,83 \\
1,84 \\
1,82 \\
1,81\end{array}$ \\
\hline $\begin{array}{l}X \ldots \\
\mathrm{R} \ldots \\
\mathrm{S} \ldots \ldots \\
\mathrm{S} \ldots \% \ldots \\
\mathrm{P}(0,95) \\
\end{array}$ & & $\begin{array}{c}1,042 \\
0,04 \\
0,015 \\
1,46 \\
+0,0204 \\
\end{array}$ & & $\begin{array}{c}1,168 \\
0,02 \\
0,0084 \\
0,72 \\
+0,0102 \\
\end{array}$ & & $\begin{array}{c}1,294 \\
0,02 \\
0,0089 \\
0,69 \\
\pm 0,0102\end{array}$ & & $\begin{array}{l}1,818 \\
0,02 \\
0,0034 \\
\quad 0,46 \\
\pm 0,0102 \\
\end{array}$ \\
\hline $\begin{array}{lll}1 & \ldots & \ldots \\
2 & \ldots & \ldots \\
3 & \ldots & \ldots \\
4 & \ldots & \\
5 & \ldots & \Xi \\
6 & \ldots & \Xi\end{array}$ & 0,73 & $\begin{array}{l}0,72 \\
0,74 \\
0,73 \\
0,76 \\
0,77 \\
0,75 \\
\end{array}$ & 1,24 & $\begin{array}{l}1,26 \\
1,27 \\
1,26 \\
1,25 \\
1,27 \\
1,26 \\
\end{array}$ & 1,36 & $\begin{array}{l}1,36 \\
1,36 \\
1,36 \\
1,37 \\
1,38\end{array}$ & 1,62 & $\begin{array}{l}1,61 \\
1,62 \\
1,61 \\
1,62 \\
1,63\end{array}$ \\
\hline $\begin{array}{l}X \ldots \\
R \ldots \\
S \ldots . . \\
S x(\%) \\
P(0,95)\end{array}$ & & $\begin{array}{c}0,745 \\
0,05 \\
0,0187 \\
2,51 \\
+0,0255\end{array}$ & & $\begin{array}{c}1,261 \\
0,02 \\
0,0078 \\
0,60 \\
+0,0102\end{array}$ & & $\begin{array}{c}1,366 \\
0,02 \\
0,0089 \\
0,65 \\
+0,0102\end{array}$ & i & $\begin{array}{l}1,618 \\
0,02 \\
0,0084 \\
\quad 0,51 \\
+0,0102\end{array}$ \\
\hline
\end{tabular}

(1) Gracieusement fourni par la Société Hoffmann-La Roche, Bâle, que nous remercions vivement. 
TABLEAU II

détermination du palmitate d'ascorbyle dans l'HUile de colza (en $\mathbf{m g} \%$ )

Additionné à l'huile de colza

Echantillons

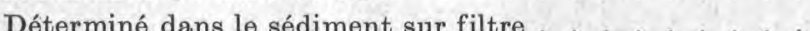

Déterminé dans l'huile de colza filtrée . . . . . . . .

La perte du palmitate d'ascorbyle . . . . . . . . .

Déterminé dans l'huile de colza après $24 \mathrm{~h}$

"

$\begin{array}{ll}\text { n } & \text { म } \\ \text { म } & \text { म }\end{array}$

i)

$$
\begin{aligned}
& 48 \mathrm{~h} .+. \\
& 72 \mathrm{~h} . \\
& 96 \mathrm{~h} . .
\end{aligned}
$$

\begin{tabular}{|c|c}
\hline I & II \\
\hline 100 & 100 \\
55 & 60 \\
25 & 20 \\
20 & 20 \\
16 & 12 \\
9 & 6 \\
traces & traces \\
0 & 0 \\
\hline
\end{tabular}

Les résultats sont rassemblés dans le tableau I, d'où il ressort que la précision de la méthode est très satisfaisante. Les plus petites quantités que l'on pouvait déterminer dans un échantillon étaient de l'ordre de 0,15 à $0,20 \mathrm{mg}$ de $\mathrm{PA}$; on obtient les meilleurs résultats avec 1,2 à $2,0 \mathrm{mg}$ de $\mathrm{PA}$ par échantillon (prise d'essai).

b) Dosage du palmitate d'ascorbyle dans une matière grasse soumise à l'autoxydation.

Pour étudier la possibilité de la détermination du PA dans une matière grasse en voie d'oxydation, on a procédé à une irradiation des échantillons d'huile de colza contenant des quantités connues de PA.

Préparation de l'échantillon : à un échantillon d'huile de colza on ajoutait 0,1 p. 100 de palmitate d'ascorbyle et on le chauffait à $45^{\circ} \mathrm{C}$ en secouant énergiquement. Au bout de $5 \mathrm{mn}$ on filtrait l'huile et on déterminait le PA dans l'huile filtrée et dans le sédiment restant sur le filtre (en solution alcoolique). Puis on laissait l'huile filtrée dans un cristallisoir à l'action de la lumière diffuse à la température de laboratoire et on en déterminait le PA tous les 24 heures. Les résultats sont montrés dans le tablèau II, d'où on voit que le PA se décompose progressivement, comme l'acide ascorbique dans une matière grasse [3]. Il semble que les produits de décomposition du PA se trouvant dans le milieu, tels que le 
composé "déhydro » et le composé "dicéto », comme cela a lieu avec l'acide L-ascorbique [5], n'ont aucune influence sur les résultats de dosage du palmitate d'ascorbyle.

\section{Summary}

There was presented a method of colorimetric determination of ascorbyle palmitate in fatty materials based on a reaction with diazotized 4-methoxy-2-nitroaniline in acid medium, folloved by development of a blue colour in alcaline solution. This colour, with a maximum absorbancy at $570 \mathrm{~m} \mu$, is compared with standard solutions in photoelectric colorimeter. The sensibility of the procedure permits to determine very minute quantities of ascorbyle palmitate. The accuracy of the method was checked by means of statistical analysis.

\section{BIBLIOGRAPHIE}

[1] A. Hотzex. Vitamine und Vitaminpräparate. Verlag W. Saenger, Berlin, 1959.

[2] R. W. Riemenschneider, J. Turner, P. A. Wells and W. C. A ult. Fatty acid monoesters of L-ascorbic and D-isoascorbic acids as antioxidants for fats and oils. Oil and Soap, 21, 47 (1944).

[3] J. BudstawSkr. Studia nad uzyciem kwasu L-askorbinowego i jego pochodnych $\mathrm{w}$ charakterze przeciwutleniaczy do masla. Zesz. Nauk. WSR w Olsztynie, 8 (60), 243 (1959).

[4] K. F. GANDER. Papierchromatographischer Nachweis von Antioxydantien. Fette-Seifen-Anstr., 57, 423 (1955).

[5] M. Schmall, C. W. Pifer and E. G. Wollish. Determination of ascorbic acid by a new colorimetric reaction. Anal. Chem., 25, 1486 (1953).

[6] M. Schmall, C. W. Pifer, E. G. Wolish, R. Duschinsky and G. GeIner. Colorimetric determination of ascorbic acid. New development concerning the reaction with diazotized 4-methoxy-2-nitroaniline. Anal. Chem., 26, 1521 (1954). 